\title{
Analysis of molecular recognition features in membrane proteins
}

\author{
I. Kotta-Loizou, G.N. Tsaousis $\bowtie$, S.J. Hamodrakas \\ Department of Cell Biology and Biophysics, Faculty of Biology, University of Athens, Athens, Greece
}

\begin{abstract}
Motivations
During the past few years there has been a growing interest in the field of intrinsically disordered proteins-related research. Intrinsically disordered proteins (IDPs) possess no rigid 3D structure under physiological conditions, yet they are functionally active. A protein may be fully or partly disordered, containing long or short intrinsically disordered regions (IDRs). Molecular Recognition Features (MoRFs), known also as Molecular Recognition Elements (MoREs), are defined as short regions that undergo disorder-to-order transition upon binding to their partners. As their name suggests, they are considered to be implicated in molecular recognition, which serves as the initial step for protein-protein interactions. Membrane proteins comprise approximately $30 \%$ of fully sequenced proteomes and they are responsible for a wide variety of cellular functions, including cell signalling. The aim of the current study was to identify and analyse MoRFs in membrane proteins.
\end{abstract}

\section{Methods}

A dataset of putative MoRFs was constructed from the Protein Data Bank, selecting membrane protein fragments between 10 and 70 residues, which interact with proteins longer than 100 residues. The assumption was made that such short aminoacid sequences would be less likely to form a rigid 3D structure prior to interaction. The initial dataset was further filtered for ambiguous information and a non-redundant dataset was created applying length-dependent thresholds. Subsequently, sequence, structural and functional analysis of the membrane MoRF non-redundant dataset was conducted.

\section{Results}

Initialy, we sought to characterize our dataset and assess membrane MoRF associations with intrinsic disorder. Approximately half of the membrane MoRFs are short, between 10 and 20 residues. In addition, membrane MoRFs' aminoacid composition was found to differ not only from that of a typical IDR, as expected, but from globular MoRFs' aminoacid preferences as well. A structure-based criterion, evaluating perresidue surface and interface areas, supported the idea that membrane MoRFs are intrinsically disordered when isolated, and undergo a disorder-to-order transition upon binding. Missing density residues, often associated with disorder, were also assessed and were found to comprise, along with irregular residues, more than half of membrane MoRFs' secondary structure. Membrane MoRFs were categorized as alpha, beta, irregular and complex, depending on their secondary structure after the interaction with their partners. Further studies were focused on MoRF-containing proteins and revealed that the vast majority are eukaryotic single-spanning transmembrane proteins. Moreover, the position of MoRFs in relation to the protein's topology was determined. Finally, functional analyses of MoRFcontaining proteins and MoRF-binding partners are currently under way. In conclusion, our goal is to provide insight into potential disorder-based protein-protein interactions involving membrane proteins. A comparison between membrane and globular MoRFs will allow us to determine if they are likewise implicated in molecular recognition procedures and whether a similar mechanism is involved. In the long term, the above information will facilitate identification, and possibly prediction, of membrane MoRFs. 